\title{
Surgery Research and Practice
}

Open Access

RESEARCH ARTICLE

\section{Complications after Totally Implantable Venous Port Catheter Intervention in Cancer Patients}

\author{
Hasan Reyhanoglu, MD* (D) and Kaan Ozcan, MD \\ Department of Cardiovascular Surgery, University Hospital of Tinaztepe, Turkey
}

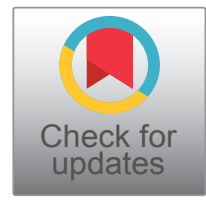

*Corresponding author: Hasan Reyhanoglu, MD, Department of Cardiovascular Surgery, University Hospital of Tinaztepe, Ahmet Piriştina Bulvarı, No: 51, Tınaztepe/BUCA-IZMiR (35390), Turkey, Tel: +90533-2401814

\begin{abstract}
Background: The aim of this study was to evaluate the complications associated with totally implantable venous port catheter interventions and the management of these complications.

Methods: Between January 2011 and October 2020, we reviewed patients who had totally implantable venous port catheter interventions. A total of 543 catheters were inserted into cancer patients via the subclavian, jugular, or femoral vein. Demographic data, venous access, primary diagnosis, catheter-related complications, catheter duration of stay, and reasons for catheter removal were retrospectively analyzed.
\end{abstract}

Results: The mean age of the patients was 53.6 years, with the sample consisting of 304 (55.98\%) females and $239(44.02 \%)$ males. The first three cancer diagnoses were breast cancer $(35.72 \%)$, colon cancer $(31.67 \%)$, and stomach cancer $(12.89 \%)$. In most patients $(72.37 \%)$, the right subclavian vein was used as the intervention site. Complications occurred in $8.8 \%$ (48 patients) of the patients in the early $(1.28 \%)$ or late periods $(6.8 \%)$ after the procedure. Catheter-related infection was the most common complication, at $2.5 \%$.

Conclusion: Some complications may occur during the insertion and use of totally implantable venous port catheters, although they are convenient for use in the treatment of patients undergoing chemotherapy. Given their long-term use in these patients, most of whom have metastatic cancer and are on immunosuppressive therapy, a careful approach during surgery and chemotherapy will reduce potential complications.

\section{Keywords}

Cancer, Chemotherapy, Total implantable venous port catheter, Complication, Oncology access

\section{Introduction}

Appropriate venous vascular access is critical in the long-term treatment process for patients diagnosed with cancer and receiving chemotherapy. Totally implantable venous port catheters (TIVPCS) are vital for easier and safer treatment delivery in these patients. These catheters, first described by Broviac and Hickman in 1973, came into use in the 1980s [1-3]. In addition to chemotherapy in cancer patients, TIVPCs are more commonly implanted because they are used in longterm intravenous therapy, blood sampling, parenteral nutrition, and blood transfusion [4,5]. The system consists of a silicone reservoir implanted under the skin and a catheter placed intravenously at the superior vena cava-right atrium junction. It is often placed via the subclavian vein or the jugular vein. However, the catheters can also be implanted via the basilic vein, brachial vein, and cephalic vein [6]. It is possible to make up to 2000 punctures after implantation if suitable needles are used $[4,7]$. The fact that the catheter lies under the skin, is invisible from the outside, and is easy to use makes chemotherapy comfortable [8]. This study evaluated the complications associated with TIVPC procedures applied to chemotherapy in oncology patients in our clinic.

\section{Materials and Methods}

Between January 2011 and October 2020, 543 patients who underwent TIVPC in our clinic were retrospectively evaluated. All procedures were carried out under operating room conditions and monitoring (electrocardiography, fingertip pulse 

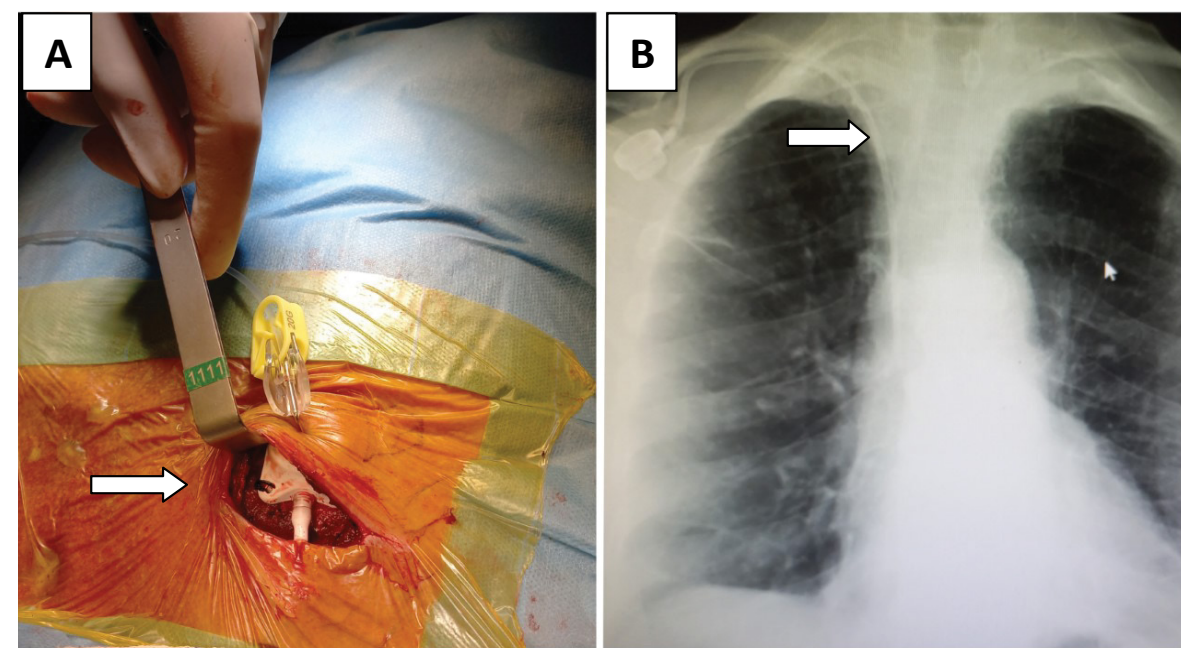

Figure 1: (A) Intraoperative view catheter after implantation; (B) View of chest X-ray image after implantation through right subclavian vein.

oximetry, noninvasive blood pressure). Hemogram and coagulation parameters were checked before the procedure. The right subclavian vein was frequently used as the intervention site and, in some patients, the jugular vein or the femoral vein. In breast cancer patients who had undergone a mastectomy, the contralateral side was used as the intervention site. All procedures were performed with local anesthesia ( $2 \%$ prilocaine hydrochloride), and additional sedation was performed in some patients. The area to be implanted was cleaned with an antiseptic solution and covered with an ioban drape. A vein puncture was performed after local anesthesia, and a guidewire was inserted. After the guidewire was seen under fluoroscopic control in the right atrium, the catheter was sent over the guidewire. The measurement was taken with the tip of the catheter at the superior vena cava-right atrium junction, and the tip was placed over the guidewire.

Subsequently, subcutaneous pocket was created 4 to $5 \mathrm{~cm}$ below the clavicle as reservoir. The reservoir was fixed under the skin and connected to the catheter (Figure 1A). Posteroanterior chest $X$-ray images were used to check the location of the catheter and potential problems (hemothorax, pneumothorax) after the procedure (Figure 1B). Following the catheter connection, the catheter was checked by puncture with a special needle (Huber needle). The reservoir and catheter were flushed with heparinized saline at the end of the procedure. A standard recommendation for non-emergency patients is to use a port catheter for no sooner than 48 hours. Antibiotic prophylaxis was not given to patients before the procedure. Celsite catheters (Celsite ${ }^{\circ}$ ST301V, B. Braun Medical, Boulogne Cedex, France) were used in 431 (79.3\%) patients, and Bard catheters (Bard, Utah, USA) were used in 112 (20.6\%) patients.

The study was carried out after obtaining the approval of the İzmir Tınaztepe University Health
Sciences Scientific Research and Publication Ethics Committee (date/number:0-04-2021/12) and detailed written consent from the patients before the procedure.

\section{Statistical Analysis}

SPSS 19 for Mac (SPSS, Inc, Chicago, II, USA) statistical program was used for statistical analysis. The data mean \pm standard deviation is reported for continuous variables, and $n$ and percentage values are used for categorical variables.

\section{Results}

The mean age of the patients was 53.6 years, with a range of $19-81$ years. A total of $55.98 \%$ of the patients were female, and $44.02 \%$ were male. The top three cancer diagnoses were breast cancer $(35.72 \%)$, colon cancer (31.67\%), and stomach cancer (12.89\%) (Table 1).

A port catheter was inserted for intravenous nutrition in 6 patients (1.1\%) with venous access problems other than a cancer diagnosis. The right subclavian vein was used in 292 (72.37\%) patients, the left subclavian vein in $141(25.9 \%)$ patients, the right jugular vein in $7(1.28 \%)$ patients, the left jugular vein in $2(0.36 \%)$ patients, and the right femoral vein in $1(1.18 \%)$ patients. The mean port catheter duration time was $352 \pm 241$ days. After the TIVPC procedure, complications developed in 48 patients (8.8\%). Complications were detected early in 7 (1.28\%) patients and late in $41(7.55 \%)$ patients (Table 2). Catheter-related infection was the most common complication (14 patients, 2.5\%). Healing with antibiotics and a local dressing was achieved in eight patients with complications, while catheter replacement was performed in six patients. The second and third most common complications were skin erosion at the reservoir site (2\%) and catheter dysfunction (1.2\%). In patients with skin erosion in which the reservoir was visible externally and there was air contact, the catheter was removed, and a new one was inserted. Catheter replacement was performed in four of six patients whose catheters could 
Table 1: Demographic and clinical characteristics of the patients.

\begin{tabular}{|c|c|c|c|}
\hline & & $N=543$ & $\%$ \\
\hline Male gender & & 239 & 44.02 \\
\hline Female gender & & 304 & 55.98 \\
\hline Age-mean & & $53.6 \pm 16.4$ & \\
\hline Age (range) & & $19-81$ & \\
\hline \multirow[t]{8}{*}{ Indications for implantation } & Breast cancer & 194 & 35.72 \\
\hline & Colon cancer & 172 & 31.67 \\
\hline & Gastric cancer & 70 & 12.89 \\
\hline & Lung cancer & 43 & 7.91 \\
\hline & Pancreatic cancer & 25 & 4.60 \\
\hline & Rectum cancer & 19 & 3.49 \\
\hline & Other malignancy & 14 & 2.57 \\
\hline & For intravenous treatment & 6 & 1.10 \\
\hline \multirow[t]{5}{*}{ Accessed vein } & Right subclavian vein & 392 & 72.37 \\
\hline & Left subclavian vein & 141 & 25.96 \\
\hline & Right Jugular vein & 7 & 1.28 \\
\hline & Left jugular vein & 2 & 0.36 \\
\hline & Right femoral vein & 1 & 0.18 \\
\hline Mean port catheter duration time-month & & $352 \pm 241$ & \\
\hline
\end{tabular}

Table 2: Post-procedure complications.

\begin{tabular}{|c|c|c|c|}
\hline & & n: 543 & $\%$ \\
\hline \multicolumn{2}{|l|}{ Early complications } & 7 & 1.28 \\
\hline & Hemothorax & 0 & 0 \\
\hline & Pneumothorax & 3 & 0.5 \\
\hline & Hematoma & 3 & 0.5 \\
\hline & Catheter migration & 1 & 0.18 \\
\hline \multicolumn{2}{|l|}{ Late complications } & 41 & 7.55 \\
\hline & Catheter-associated infection & 14 & 2.5 \\
\hline & Catheter dysfunction & 6 & 1.2 \\
\hline & Catheter-reservoir disconnection & 1 & 0.18 \\
\hline & Septic embolism & 1 & 0.01 \\
\hline & Reservoir torsion & 4 & 0.7 \\
\hline & Skin erosion & 11 & 2.0 \\
\hline & Venous thrombosis & 4 & 0.7 \\
\hline \multicolumn{4}{|c|}{ Complications by intervention site } \\
\hline & Right & 35 & 6.4 \\
\hline & Left & 13 & 2.3 \\
\hline \multicolumn{4}{|c|}{ Complications by gender } \\
\hline & Male & 21 & 3.8 \\
\hline & Female & 27 & 4.9 \\
\hline \multicolumn{4}{|c|}{ Complications by catheter brand } \\
\hline & Celsite catheter ${ }^{*}$ & 34 & 6.2 \\
\hline & Bard catheter $^{* *}$ & 14 & 2.5 \\
\hline \multicolumn{2}{|l|}{ Total complications } & 48 & 8.8 \\
\hline \multicolumn{2}{|l|}{ Mortality } & 0 & 0 \\
\hline
\end{tabular}

${ }^{\star}$ Celsite ${ }^{\circledR}$ ST301V, B. Braun Medical, Boulogne Cedex, France); “*Bard catheters (Bard, Utah, USA) 
not be used because of catheter dysfunction, and new catheters were not inserted in two patients. In all patients who underwent catheter replacement, a different venous access side was preferred as the new catheter access, often the contralateral subclavian vein access side. Pneumothorax developed in three patients during the procedure, two of whom required tube thoracostomy, while minimal pneumothorax regressed spontaneously in one patient, and tube thoracostomy was not needed. In three patients who developed a hematoma on the reservoir side, the hematoma was evacuated by puncture. In four patients who developed venous thrombosis in the upper extremity (subclavian or jugular), the catheter was removed, and treatment with low molecular weight heparin (LMWH) was started. The catheter was urgently removed from one patient due to a septic embolism with fever. Staphylococcus aureus was detected on a culture antibiogram, and the patient was started on appropriate antibiotics; the catheter was not replaced with a new one. A patient with catheter migration was taken to the emergency cardiology laboratory, where the catheter was removed using a snare from the right atrium. Reservoir torsion developed in $4(0.7 \%)$ patients. In patients in whom torsion was noted during chemotherapy, the subcutaneous tissue was opened, and the reservoir was fixed in the appropriate position. In one patient with catheter disconnection, subcutaneous swelling developed during chemotherapy, and the connection was restored by incising the reservoir area. Complications developed in $6.2 \%$ of patients with the Celsite brand and $2.5 \%$ of patients with the Bard brand catheter. Since $73.4 \%$ of the interventions were performed on the right side, complications were also detected more frequently on the right side (6.4\% vs. $2.3 \%)$. No major cardiovascular bleeding, severe arrhythmias, or mortality occurred in the patients (Table 2).

\section{Discussion}

Totally implantable venous port catheters (TIVPCS) provide great convenience in the administration of treatment in patients under chemotherapy. These catheters have become increasingly preferred in recent years because of phlebitis, cellulitis, and skin necrosis due to damage from drugs used in chemotherapy and extravasation in peripheral veins [9]. The main advantages of these catheters are that they are easy to implant, not visible from the outside, do not cause discomfort, are easy to puncture, and have a low risk of infection $[4,10]$.

Despite these advantages, some complications may occur with the insertion and use of these catheters because they are also used for frequent blood sampling, parenteral nutrition, intravenous therapy, and the administration of chemotherapy in oncological patients in long-term treatment processes. Due to these additional uses, catheter occlusion, extravasation, and reservoir site complications are more common.
The literature has indicated hemothorax, pneumothorax, hematoma, arrhythmia, cardiac perforation, embolism, damage to the thoracic duct, damage to the brachial plexus, and malpositioning as complications observed in the early stage of catheter instalment. In the late stage, complications such as skin necrosis or erosion, infection, septic embolism, disconnection, reservoir torsion, catheter-related venous thrombosis, and fluid extravasation may occur $[4,5,10]$. The overall complication rate associated with TIVPC procedures has been reported in the literature to be $3.1-33.9 \%[5,11]$. In our series, this rate was $8.8 \%$, and catheter-related infection was the most common complication (2.5\%). The incidence of catheter-related infections has been reported as $0.6-27 \%$ in the literature $[8,11]$. Infection may occur at the reservoir site, along the catheter line, and in the form of catheter-associated bacteremia [8]. The most common bacterial types in blood cultures for this type of infection have been reported to be Staphylococcus aureus, Staphylococcus epidermidis, Klebsiella spp., Escherichia coli, and Candida albicans [6]. The risk of infection is increased by the presence of cancer, immunosuppressive therapy, some types of cancer (hematologic and gastrointestinal), frequent hospitalization, the use of drugs other than chemotherapy, and frequent punctures $[5,9]$. In cases of local infection of the needle puncture site without bacteremia or fever, local dressing and antibiotic therapy may sometimes be sufficient, whereas in the presence of symptoms such as wound growth and fever, removal of the catheter should be the appropriate course of action $[5,12]$. If insertion of a new catheter is planned, it is reasonable to insert it from a different site and postpone the procedure until the bacteremia has been eliminated.

The rate of catheter-related venous thrombosis reported in the literature is $5-40 \%$ [4]. Venous thrombosis often occurs along the vein into which the catheter is inserted. This risk is higher in patients over 65 years of age with a history of deep venous thrombosis, patients with multiple comorbidities, and those with certain cancers (lung, gastrointestinal) [8]. In addition to the catheter itself being the cause of thrombosis, hypercoagulability due to malignancy, some drugs used in chemotherapy (5-fluorouracil, interleukin-2), previous surgical procedures, and bed rest increase the risk of thrombosis $[4,6,12]$. Further, polyurethane catheters have been reported to cause less thrombosis than polyvinyl chloride, tetrafluoroethylene, and polyethylene catheters [12-14]. Some studies have reported that the insertion site of the catheter is an influential factor in thrombosis, and left-sided catheters cause more thrombosis [15]. In this study, thrombosis developed on the right side in all four patients who developed venous thrombosis, and LMWH was started in all of them after removing the catheter. Removal of the catheter and the start of anticoagulant therapy 
should be the first choice in this case, as frequent pain and swelling in the arm or neck can occur [5]. Although studies have reported 5-40\% pulmonary embolism due to venous thrombosis, no pulmonary embolism was observed in our patients [10].

The most feared early complication of catheterization, pneumothorax, has been reported in the literature at an incidence rate of 1.5-6\% [8]. Pneumothorax necessitates a lengthy hospital stay and therefore presents as the most traumatic complication for patients. This risk has been reported to be high, especially in patients who have had more than one puncture; therefore, ultrasound-guided puncture is used to reduce this risk [15]. Two of the three patients who developed pneumothorax required tube thoracostomy in our case, while one patient's minimal pneumothorax regressed during the 24-hour follow-up and did not require tube thoracostomy. It should be noted that pneumothorax may be overlooked in preoperative fluoroscopy imaging. Therefore, checking with chest X-ray is critical to detect pneumothorax and other early complications before the patient is discharged a few hours after the procedure [8].

One of the complications that we did not encounter in our study but that has been reported in the literature is "pink-off" syndrome $[10,16]$. Lin, et al. reported catheter fractures associated with this syndrome in 73 (2.1\%) patients in their series of 3358 patients [16]. After the catheter is compressed through the clavicle and $1^{\text {st }}$ rib, symptoms such as arm swelling, infraclavicular pain, resistance to infusion, and arm paresthesia may develop in this syndrome [5]. A ruptured fragment might sometimes result in pulmonary embolism or cardiac complications [4].

Catheter dysfunction and migration are other common complications. The most common reason is that catheters are often used in blood sampling and are not flushed with heparinized saline afterward. After blood sampling, the blood remaining in the catheter forms a plug in the catheter [17]. Another reason is the position of the catheter in the vein. It is critical to fix the catheter at the superior vena cava-right atrial junction to prevent catheter dysfunction $[7,8]$. The distal intracardiac location causes rhythm problems, whereas the jugular and brachiocephalic positions increase the risk of dysfunction $[5,17]$. Antegrade infusion is sometimes possible, although no blood is aspirated during catheter application. This is due to the catheter sticking to the vessel wall during negative pressure or a fibrin layer covering the catheter tip [4]. Catheter migration often develops during the procedure. This complication, which often leads to dramatic results, occurred in one patient in our series. The catheter was removed from the right atrium using a snare through a femoral vein in the angiography laboratory.

Another complication is skin necrosis at the reservoir site. The most common causes are frequent punctures, medication irritation, sharp-edged port catheters, and skin thinning due to weight loss over time. This problem is widespread in end-stage cancer patients. The placement of the reservoir deeper under the pectoralis fascia in such patients may prevent this complication [17].

Reservoir torsion was another complication we encountered in our series. This complication, which usually occurs after frequent manipulation of the reservoir, developed in four patients. The subcutaneous tissue was opened in all patients, and the reservoir was placed in the proper position. Reservoir fixation was performed with absorbable sutures (3/0 Vicryl) in all of these patients. For this reason, we still performed reservoir fixation sutures with non-absorbable suture material ( $3 / 0$ silk).

In addition to placing these catheters through the jugular and subclavicular veins, it is also possible to insert them through the brachial and femoral veins. However, the use of the brachial vein and femoral vein increases the risk of thrombosis due to the bending of the arm due to arm movements and the greater length of the catheter [17].

In recent years, with the increase in chemotherapy applications, TIVPC procedures have also increased. Therefore, various departments (anesthesiology, cardiovascular surgery, general surgery, thoracic surgery, interventional radiology) perform this procedure, which can be a problem in many centers. We believe that TIVPC procedures should be performed by a department that has the expertise to overcome the complications associated with these procedures.

The limitations of our study are that it was a retrospective and non-randomized study. Our study was designed based on our experiences with these interventions. Consequently, TIVPC procedures should be performed carefully to avoid early complications during the implantation. Considering that these patients are usually under immunosuppressive treatment, maximum attention to sterility during the procedure and flushing at regular intervals with heparinized saline can reduce the number of late complications. To this end, training the staff administering chemotherapy will extend the useful life of these catheters.

\section{Conflict of Interest Statement}

The authors declare that there is no conflict of interest during the study or authorship of this article.

\section{Funding}

During the study and the authorship of this study, the authors declared that they did not receive any financial funding.

\section{References}

1. Broviac JW, Cole JJ, Scribne BH (1973) A silicone rubber 
atrial catheter for prolonged parenteral alimentation. Surg Gynecol Obstet 136: 602-606.

2. Hickman RO, Buckner CD, Clift RA, Sanders JE, Stewart $P$, et al. (1979) A modified right atrial catheter for access to the venous system in marrow transplant recipients. Surg Gynecol Obstet 148: 871-875.

3. Niederhuber JE, Ensminger W, Gyves JW, Liepman M, Doan K, et al. (1982) Totally implanted venous and arterial access system to replace external catheters in cancer treatment. Surgery 92: 706-712.

4. Üstüner MA, İlhan E, Zengel B, Telciler KE, Ertürk Ö, et al. (2013) Using of venous port catheter in patients with cancer: A 5-year clinical experience. Medical Journal of İzmir Hospital 17: 198-205.

5. Velioğlu Y, Yüksel A, Sınmaz E (2019) Complications and management strategies of totally implantable venous access port insertion through percutaneous subclavian vein. TJTCS 27: 499-507.

6. Campagna S, Gonella S, Berchialla P, Morano G, Rigo C, et al. (2019) Can peripherally inserted central catheters be safely placed in patients with cancer receiving chemotherapy? A Retrospective Study of Almost 400,000 Catheter-Days. Oncologist 24: 953-959.

7. Walser EM (2012) Venous access ports: Indications, implantation technique, follow-up and complications. Cardiovasc Intervent Radiol 35: 751-764.

8. Machat S, Eisenhuber E, Pfarl G, Stübler J, Koelblinger C et al. (2019) Complications of central venous port systems: A pictorial review. Insights Imaging 10: 86.

9. Yanık F, Karamustafaoğlu YA, Karataş A, Yörük Y (2018) Experience in totally implantable venous port catheter: Analysis of 3,000 patients in 12 years. TJTCS 26: $422-428$.
10. Kaygın MA, Dağ Ö, Güneş M, Şenocak M, Erkut B (2012) The use of intravenous port in maling disease: 5-year experience. Selcuk Medical Journal 28: 17-21.

11. Yildizeli B, Laçin T, Batirel HF, Yüksel M (2004) Complications and management of long-term central venous access catheters and ports. J Vasc Access 5: 174178.

12. Ballarinia C, Intraa M, Cerettia AP, Cordovanaa A, Pagania $M$, et al. (1999) Complications of subcutaneous infusion port in the general oncology population. Oncology 56: 97-102.

13. Lin WY, Lin CP, Hsu CH, Lee YH, Lin YT, et al. (2017) Right or left? Side selection for a totally implantable vascular access device: A randomised observational study. $\mathrm{Br} \mathrm{J}$ Cancer 117: 932-937.

14. Shivakumar SP, Anderson DR, Couban S (2009) Catheterassociated thrombosis in patients with malignancy. J Clin Oncol 27: 4858-4864.

15. El-Balat A, Schmeil I, Karn T, Holtrich U, Risteska LM, et al. (2018) Catheter-related complications of subcutaneous implantable venous access devices in breast cancer patients. In Vivo 32: 1275-1281.

16. Lin $\mathrm{CH}$, Wu HS, Chan DC, Hsieh CB, Huang $\mathrm{MH}$, et al. (2010) The mechanisms of failure of totally implantable central venous access system: Analysis of 73 cases with fracture of catheter. Eur J Surg Oncol 36: 100-103.

17. Özer AB, Bayar MK (2011) Review of our implantable venous port catheter applications. FMJ 16: 6-10. 